\title{
Alter ist nicht allein entscheidend
}

\section{Vorstand der SGGA}

Schweizerische Gesellschaft für die Gesundheit Adoleszenter

\section{Positionspapier - Zusammenfassung*}

- Kinderkliniken in der Schweiz sollen die ambulante und stationäre Behandlung für alle Jugendlichen bis mindestens 18 Jahre anbieten.

- Der Transitionsprozess von Jugendlichen von der Pädiatrie in die Erwachsenenmedizin soll frühzeitig geplant werden. Der allein vom chronologischen Alter abhängige Transfer von Jugendlichen in die Erwachsenenmedizin soll aufgegeben werden. Stattdessen sollen Selbst-Management-Fähigkeiten des jugendlichen Patienten sowie seine Kompetenz in der Interessenwahrnehmung gegenüber dem Behandlungsteam ausschlaggebend sein für die Beurteilung der Bereitschaft für einen Transfer.

- Fachpersonen der Erwachsenenmedizin, die über wenig Erfahrung in der Behandlung von jugendlichen Patienten verfügen, sollen in ihren Bemühungen unterstützt werden, Jugendliche und junge Erwachsene altersangemessen und umfassend unter Berücksichtigung biopsychosozialer Entwicklungsaspekte zu betreuen.

- Gewisse Patienten mit angeborenen seltenen Krankheiten benötigen unter Umständen eine LangzeitZusammenarbeit zwischen den pädiatrischen Spezialisten und dem erwachsenenmedizinischen Behandlungsteam bis weit über 18 Lebensjahre hinaus.

\section{Stellungnahme der SGIM - Zusammenfassung*}

Eine Erhöhung der Altersgrenze für den Zugang zu kinder- und jugendmedizinischen Diensten auf 18 Jahre ermöglicht eine flexiblere ambulante und stationäre Betreuung Jugendlicher sowie die Berücksichtigung der körperlichen, psychischen und sozialen Entwicklung auch junger Erwachsener.

Die Ausbildung medizinischer Fachkräfte ist von zentraler Bedeutung und sollte sowohl Kinder- und Jugendmedizinern als auch Allgemeinmedizinern, Allgemeininternisten, Spezialisten der Inneren Medizin und im stationären Bereich tätigen Internisten gemeinsame Schulungsmassnahmen anbieten, auf deren Basis sie gemeinsam Betreuungskonzepte entwickeln können.

Ein Übergangsplan sollte sowohl Jugendlichen, die im Rahmen der Kinder- und Jugendmedizin betreut werden, als auch Jugendlichen über 16 Jahren im Anfangsstadium einer chronischen Erkrankung, die im Rahmen der medizinischen Versorgung Erwachsener betreut werden, vorgeschlagen werden.

Unser Dank gilt den Dres. Anne Meynard, Dagmar M. Haller-Hester und Françoise Narring, Universitätsspital Genf, für ihren Beitrag zu dieser SGIM-Stellungnahme und Prof. Valérie Junod, Fakultät für Rechtswissenschaften der Universität Genf, für ihre kritische Überarbeitung und Hilfe bei rechtlichen Fragen.
Die ausführlichen Versionen finden sich unter www.saez.ch $\rightarrow$ Aktuelle Ausgabe oder $\rightarrow$ Archiv $\rightarrow 2015 \rightarrow 44$

Korrespondenz: Geschäftsstelle SGGA Haldenrain 18 CH-8909 Zwillikon Tel. 0794496206 info[at]sgga-assa.ch

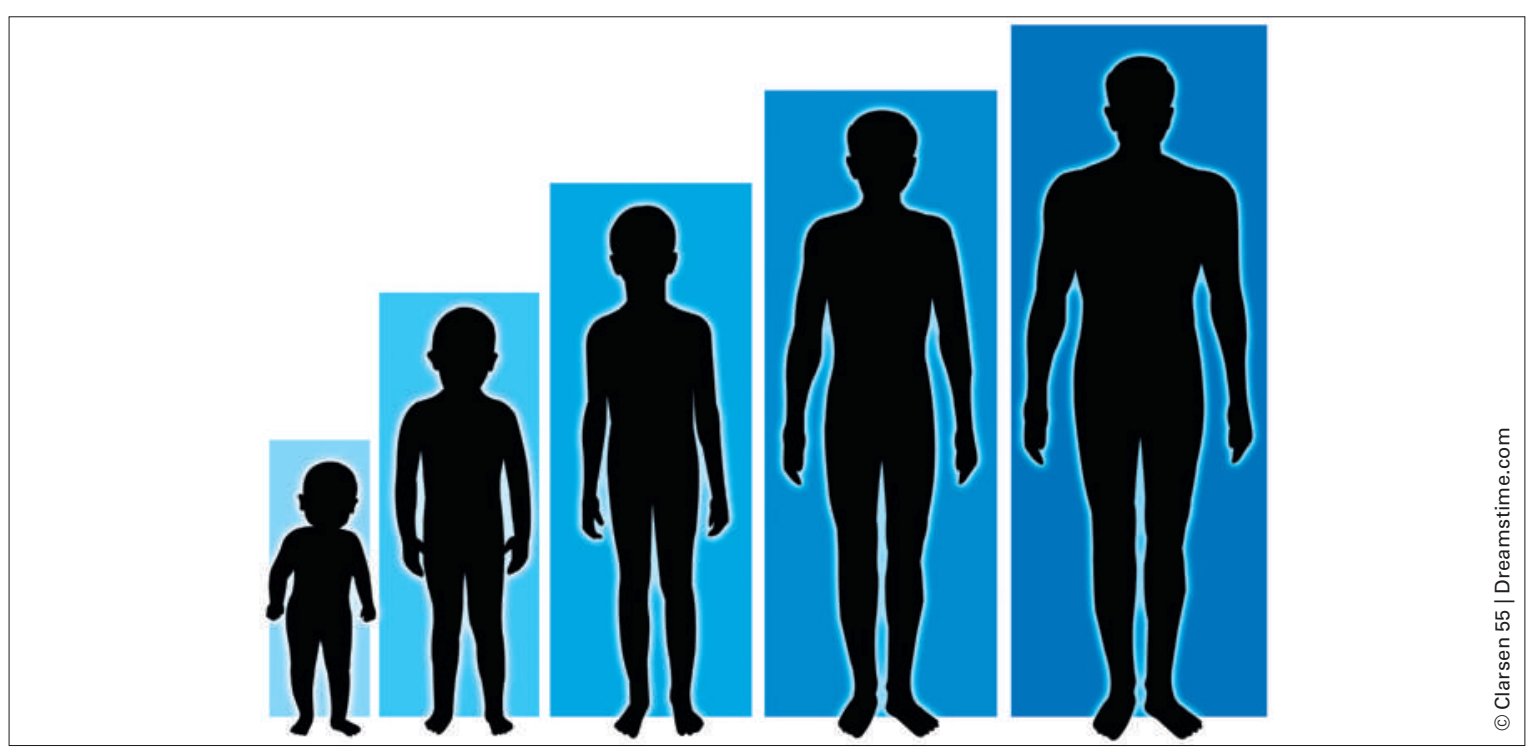

Der allein vom chronologischen Alter abhängige Transfer von Jugendlichen in die Erwachsenenmedizin soll aufgegeben werden. 